\title{
Artificial Intelligence in Health Care and Medicine: A Personalized Approach
}

\section{Koen V Hindriks ${ }^{1}$ and John-Jules Ch Meyer ${ }^{2 *}$}

${ }^{1}$ Vrije Universiteit Amsterdam, The Netherlands

${ }^{2}$ Utrecht University, Utrecht, The Netherlands/Emotional Brain, Almere, The Netherlands

*Corresponding Author: John-Jules Ch Meyer, Utrecht University, Utrecht, The Netherlands/Emotional Brain, Almere, The Netherlands.

Received: August 06, 2019; Published: September 19, 2019

DOI: 10.31080/ASMS.2019.03.0410

\begin{abstract}
Artificial Intelligence (AI) is becoming more and more ubiquitous, and AI also invades health care and related fields ever more. We believe this is a good thing. Inspired by work that we have done in various projects at the Alan Turing Institute Almere (ATIA), Delft University of Technology (TUD), Utrecht University (UU), and Vrije University (VU), we'll review a number of applications of AI in health care and medicine, mainly from a personal viewpoint, expertise and interest. In many of the projects we have worked on there is a shared focus and interest in creating a more personalized approach to health care and medicine. It is this focus on a more personalized health care that we want to highlight in this paper. To this end, we review various examples of work to illustrate and argue that AI, and Social AI in particular, has great potential for improving healthcare and enabling a more personalized approach. We show techniques from Social AI can be effectively applied in developing Behavior Change Management systems, and illustrate its use in related work on data collection for Value-Based Health Care. In particular, we'll discuss artificial emotions, serious games for healthcare, and artificial companions to assist with the care of patients in a hospital or home setting. These companions are able to monitor the behavior of patients, help them remind of taking medication, but also can have conversations with them giving them the feeling that they are cared for. We argue that these examples of Social AI techniques can be used to enable and improve personalized health care even in times where health care is economized upon like we face in The Netherlands. More generally, in this article we discuss various methods and techniques from AI to do this.
\end{abstract}

Keywords: Artificial Intelligence; Health Care; Medicine

\section{Introduction}

Artificial Intelligence (AI) has gained a lot of interest in health care and medicine more recently. AI techniques have been applied in very diverse domains of health care including drug discovery, medical imaging, personalized genetics, robots in surgery rooms, and providing preventive support for elderly independent living. Advanced machine learning techniques have been used to diagnose cancer from images, for example, and social robots have been used to motivate children with diabetes to adhere to a lifestyle regime. Both authors have been involved in several projects in which $\mathrm{AI}$ has been applied to improve health care. The first author has been involved in projects, for example, in which social robots have been developed to improve lifestyle management for diabetes, to reduce stress of children with cancer, and to implement Value-Based Health Care (VBHC) by automating the collection of patient-reported outcome measures by means of an interviewing robot. The second author has been involved as a scientific director at the Alan Turing Institute Almere (ATIA), where data science, data analytics, and machine learning techniques have been used to develop health and medicine applications, together with several other institutes, as well as with academic hospitals. A shared focus in all of these projects has been the use of (Social) AI techniques for 
developing a more personalized approach in health care and projects that have aimed at improving the daily practice of care in realworld contexts such as hospitals. Thus we show how we can use several methods and techniques from AI, and particularly Social $\mathrm{AI}$, to address problems in health care in a personalized manner. We will explain the problems and solutions used per topic. It is important to note that there is not just one method or technique that is used but there are various quite distinct ones that can be employed. The methods/techniques/solutions range from employing emotional models to multi-agent systems and from data analytics to employing social robots.

The paper is organized as follows. We first introduce Social AI and discuss its potential and application in health care. We discuss themes such as gamification of health care training, artificial emotions, and the potential of social robots to improve health care. Then we continue to discuss work that aims at behavior change and provide examples for lifestyle management and mental health. Finally, we present our conclusion and briefly summarize the potential of (Social) AI for personalized health care as we see it.

\section{Social AI in Health Care}

Social AI aims to improve how AI systems understand and interact with people. Common examples of such systems are chatbots that use natural language for interfacing with people, virtual assistants and virtual reality applications, smart speakers and displays that use voice to interact with people, and social robots that provide yet another interface for interacting with their users. In the health care domain, all of these different types of interfaces can be used to achieve a variety of objectives. Social AI techniques can be used to improve patient engagement and experience, to personalize health care interventions, and to assist with the training of health care professionals. We will illustrate this potential of AI by discussing several example projects that we have been involved in one way or another below. We also argue that multi-agent systems, that consist of multiple artificial agents that interact with each other, have potential and can provide an interesting new perspective on personalized medicine.

Serious games for health care training and independent living

Serious games can be used as virtual training environments, and provide an innovative approach to training where Social AI is used to create realistic training scenarios. Typically, these games are simulations of situations that are useful for practicing various skills. In a serious game, the trainee plays a role and interacts with so-called non-player or virtual characters. The idea of these serious games arose from economizing real simulations where situations were enacted by a lot of people in a staged real-world setting. This is costly and sometimes impossible if the situations at hand are rare or dangerous. The virtual characters in serious games are controlled by intelligent agent software [31], and the main thing is that they must be believable in order for the trainee to think the whole simulated situation is real enough to have a learning experience. Nowadays these serious games are ubiquitous, and have many applications, for example for firemen, police men and military. But there are also serious games for health care [3] which can be used to train health care professionals. We have worked on the personalization of this type of games involving automatic adaptation to the knowledge and skills of the user/trainee.

A feature that is very important in serious games is explain ability. It is crucial, for example, that a trainee who is engaging in a serious game can understand why other characters are behaving the way they do and why they take certain decisions or provide a certain advice. One of the very interesting things is that virtual characters in serious games, especially in health care, must deal with emotions to some extent, which is the subject of the next section.

Another illustration of the use of serious games in health care is the use of such a game to support independent living for elderly by improving the skills of elderly for handling scams. Elderly people that live at home face many issues, one of which is falling prey to so-called doorstep scams. In situations where unadvertised people present themselves at the door of the homes of elderly, many of the elderly find it difficult how to handle these situations and to differentiate bona fide people from doorstep scammers. Training of these situations can help improve their skills and make them more assertive towards these scammers [26,27] report on an agent-based training environment that aims to improve the verbal resilience of elderly when faced with doorstep scams. They use a virtual environment to simulate doorstep scam situations and ask trainees to select the most appropriate response. The system uses speech recognition to detect the level of assertiveness and provides immediate feedback to the user's performance. 


\section{Artificial emotions}

A relatively new development in $\mathrm{AI}$ is the study of (artificial) emotions. In a way this is just a continuation of studying the (computer simulation of the) human mind which was first entirely focused on cognitive behavior and reasoning. But due to developments in psychology and the humanities, emotions have become a serious topic of investigation and we have developed formal models of emotions that can be used in serious games discussed above and by social robots which we will discuss below. The aim is to put these formal models at work in systems where (emotional) software agents steering virtual characters or robots can render these more believable. This is particularly important for application in health care and to support a more personalized treatment of patients. As we shall see later in this article emotions play an important role not only in serious games for health care as mentioned earlier, but also in robot-patient systems to assist patients in health care situations.

In former days emotions were seen as irrational behavior disturbing the rational mind. But since neurologist Damásio [1], this has changed radically: emotions are now viewed as complementary to rational thinking and behavior, and in support of these. And so it made sense to use these insights also for artificial intelligence, and thus the discipline of affective computing entered the field [38]. Models of emotions are not only useful for getting artefacts with a more human-like behavior, but also play a functional role. As was recognized by psychologists like Frijda, "Emotional states organize ready repertoires of action" and "Emotions are heuristics", e.g., [8]. So emotions may help organize behavior of agents. Speaking in terms of software engineering, emotions may act as a design tool for the software construction of artificial agents such as chatbots, virtual characters, or robots. This is the perspective we took when we started to investigate emotions [30,48-52]. In a series of papers, we have investigated models of emotions; we mostly started with logical formalizations of psychological models after which we tried to make these into computational models with the aim to get computer-based systems that deal with emotions in some way.

The methodology that we applied is called the synthetic method in the literature $[17,47]$. It comprises the following steps in realizing intelligent socio-technical systems:
- $\quad$ Observing (social) phenomena

- Theory construction

- Pre-formal theory

- Formal characterization of (social) concepts

- (Logical) calculus

- Development of a computational model

- Principled operationalization

- Computer model of artificial system

- Artificial system

- Observed performance

This is in fact what we did: we started out with a psychological theory based on observations such as Oatley and Jenkins [35] and OCC [36], which we formalized in a specific type of modal logic (i.e., KARO [32]), after which we derived computational models (cf. [41]) from this work, mostly using the programming language 2APL [2]. As we shall see these computational models of emotions can be used proficiently in several health care systems such as serious games and robotic assistance systems.

\section{Decision support and medical recommendations}

Another subfield related to healthcare and medicine, where AI researchers have also played an important role is in obtaining computer-based medical practice guidelines development. We have not been involved in any work in this area ourselves but interesting work in this area is $[9,53,54,56]$ who develop evidence-based clinical practice guidelines for promoting standards of medical care. Clinical practice guidelines are recommendations for clinicians about the care of patients with specific conditions. They should be based upon the best available research evidence and practice experience. We believe this line of research is interesting and important and has potential.

\section{Social robotics}

Introducing robotics in healthcare can compensate the shortage in the human workforce. Robots can offer more time for health care professionals to provide care rather than deliver items or spend their time on administrative tasks. Surgery robots can be used in the surgery room, operated by medical specialists, to provide precision surgery, and mobile delivery robots can be used to deliver medications and transport sterile surgical supplies or linen. A different kind of robots, called social robots, equipped with touch sensors, cameras, and microphones, have been designed for interac- 
tion with people. These robots can operate autonomously, that is, without human intervention, and in a health care context can be used to alleviate loneliness of the elderly, support medication adherence for patients with chronic diseases, and to reduce stress while undergoing medical treatment, for example. We illustrate some of these applications in this section and the next.

Our first example illustrates how social robots can help bring about new approaches to health care such as Value-Based Health Care (VBHC). Instead of rewarding volume, VBHC aims to reward better results in terms of cost, quality, and outcome measures. Measuring patient outcomes is essential for the success of valuebased health care. To this end, so-called Patient Reported Outcome Measures (PROMs) are used which enable a data-driven health care approach for assessing quality of life. This approach also supports physicians and nurses in delivering personalized health care. However, the administration of PROMs requires considerable effort and puts a large burden on today's healthcare systems. To alleviate this burden, we have proposed the use of the social robot Pepper from Softbank for cost-effective robot-mediated administration of PROMs. An initial prototype of a PROM-interview robot has shown great promise and we were able to show data can be reliably collected by a robot $[4,5,13]$, but some challenges that we are working on remain. First, there is a need for more advanced patient-robot dialog, interview, and explanation capabilities that, for example, allows patients to elaborate on their answers when they want to. Second, as patients questioned about their wellbeing may respond emotionally, the robot needs to be able to weigh social signals indicating frustration and patient stress and take their emotions into account. Third, as the needs of individual patients are different and patients repeatedly visit the hospital, more personalized robot interaction is needed that enables the robot to adapt its behavior to specific patient characteristics and previous encounters.

Social robots can be particularly effective and motivating for children in various contexts. In our second example we discuss a project that aims to reduce stress of children with cancer. The aim of the project is to contribute to the development of a robot that is able to facilitate child-robot bonding [22]. Work has been done to build such bonds by providing the robot with the ability to conduct a getting acquainted interaction [23] and to engage in interactive storytelling where the child plays a role in how the story unfolds and participates by physically enacting parts of the story together with the robot. We aim to use robot-disclosure and a shared interaction history to make the child feel comfortable enough to talk about their day with the robot. This requires new forms of interaction design and AI for social interaction. Apart from these technical challenges, one of the other challenges in the project has turned out to be to get access to the hospital children and deploy the robot.

In all of these examples emotions play an important role in the interaction between robot and patient. We believe that the emotion models we discussed above can also increase the efficacy of human-robot interaction. Emotions are also important more broadly in human-computer interaction [33]. Nowadays we see several initiatives for including emotions in companion robots $[45,46]$. This is an important development for health care ([45], but for a more critical perspective, see [7]).

\section{Multi-agent systems for personalized medicine}

The second author was also involved in the development of AI techniques, such as multi-agent systems, machine learning, and knowledge-based reasoning, for multidisciplinary health research to individualise disease treatment. We adhered to the paradigm of personalized medicine ${ }^{1}$ and tried to retrieve/infer as much information about an individual as possible. That is, we tried to find and exploit differences and similarities, using combinations of characteristics to achieve:

- Treatment efficacy: group vs. individual (Responders vs. Non-responders) Example: Depression study

- $\quad$ Subtyping to improve treatment (Same diagnosis does not imply same cause) Example: Obesity study

- $\quad$ Find new Patterns and links

And we could answer questions such as:

Which combination(s) of characteristics can predict: diagnosis, treatment efficacy and adverse events.

${ }^{1}$ https://en.wikipedia.org/wiki/Personalized_medicine 
We used a so-called multi-agent approach to assemble a toolset of various distinct techniques to be used on the projects at hand, where sometimes even more than one technique could be used in parallel. The multi-agent system consisted of several modules (agents), each with their own knowledge representation and/or reasoning methods, and provided an 'Inter-agent dialogue platform'. The modules (agents) comprised machine learning for inductive reasoning using decision trees (these trees resulted in rules that then could be used in deduction) [40] and artificial neural networks (Restrictive Boltzmann Machines [20]), mutual interaction information between variables [43], Bayesian networks [37], rule-based reasoning (deduction using KR rules, [6]), regression analysis [24] and k-means clustering [25]. An example of our work was published in [14]. We believe this is one of the first works to use this multi-agent technique, which seems to have become more fashionable nowadays (see e.g. [44]). The system can be used for diagnosis and advice for complex multidisciplinary domains, using multiple independent lines of reasoning, and obtaining statistically more reliable results.

A main problem with machine learning techniques is a lack of transparency, which causes the fact that results are badly explainable, even to experts, but certainly to laymen... Explainability, like for serious gaming, is therefore an important feature such techniques should have. One way of trying to remedy this transparency problem in machine learning is to use several distinct techniques in parallel as in [14]: observing similar results by using distinct techniques can provide more confidence in the results, and if one of the techniques employed is of a more symbolic/logical nature, this may provide explanations of the results in a way that is intelligible for a human.

\section{Behavior change management}

Health care faces many problems these days such as an increasingly large elderly population which requires more care while there is a lack of resources to do so. Because of these issues prevention of, for example, chronic and other diseases or health care related phenomena (e.g., diet) by means of improving lifestyle has become an important focus. Behavior change management systems can be applied to deal with many of these issues. Like with the interview- ing robot, behavior change techniques can be used to reduce costs and workload by shifting some of the work to AI systems.

Behavior change systems have been applied to address lifestyle in relation to various health care issues. The PROMISS project aims to improve active and healthy ageing by preventing protein energy malnutrition in seniors ${ }^{2}$. In Europe, between $13.5 \%$ and $29.7 \%$ of older adults living at home are malnourished or at risk of protein energy malnutrition [28] introduces a system with persuasive communication to improve the diet compliance and adherence of participants in the PROMISS-project. Different strategies for personalization are used, taking framing, politeness, and persuasion principles into account. The design of the system, moreover, is tailored to the specific needs of elderly. One of the most important lessons learned during the development of the system is that both the system as well as the participants need to have some room for flexibility [28]. Interaction with technology is a two-way street that requires the technology to provide room for users to do things as they are used to and enjoy. But it also requires users to adapt to the technology they interact with.

Another example concerns the application of behavior change technology to treat insomnia [15] gives an overview of persuasive strategies to derive high-level requirements for a virtual sleep coach. It finds that most persuasive strategies can be used for developing a virtual sleep coach but the challenge is how to combine persuasive strategies and how to determine when to apply a particular strategy. In [16] a fully automated mobile phone app that applies cognitive behavioral therapy for insomnia is evaluated in an RCT. It demonstrates the efficacy of the app in the treatment of relatively mild insomnia. Although this technology shows great promise, it remains a challenge how to best integrate this kind of technology into a health regime.

In our final example we again discuss social robots to show that they do not only provide mere companionship to children and elderly [34], but also can help in maintaining certain types of life style and accompanying medical treatment [19,29,39]. They can help people and particularly children with dealing with several kinds of diseases such as autism $[21,42,55]$ and diabetes $[11,12]$.

${ }^{2}$ https://www.promiss-vu.eu/

Citation: Koen V Hindriks and John-Jules Ch Meyer. "Artificial Intelligence in Health Care and Medicine: A Personalized Approach". Acta Scientific Medical Sciences 3.10 (2019): 71-78. 
Type 1 Diabetes Mellitus (T1DM) in children requires a high level of self-management of young patients. In the Personal Assistant for healthy Lifestyle (PAL) project, a social robot and its (mobile) avatar have been developed which can act as a child's pal or companion. When patients do not acquire the knowledge, skills and habits to adhere to their diabetes regimen at childhood, risks associated with diabetes increase at adolescence. The PAL-project has assessed the benefits of the behavioral change on patients' health conditions, and the profits for caregivers in longitudinal field experiments. One interesting new module that was developed in the project, again highlighting the importance of emotion, enables agents to use emotions to (self-)explain their actions [18]. This gives rise to Emotion-aware eXplainable Artificial Intelligence (EXAI) [18]. argues that simulating emotions using appraisal theory enables (1) the explanation of these emotions, (2) the use of emotions as a heuristic to identify important beliefs and desires for explanation, and (3) the use of emotion words in the explanations themselves.

\section{Conclusion}

In conclusion, we dare say that AI, and particularly Social AI, holds a big promise to health care and medicine. It will provide for more advanced and easy-to-use techniques, both in the clinical environment and in common daily health care and medicine. Thus, we will see more AI in health care and medicine in the years to come, at least along the lines we've sketched in this short article. In our opinion this is a good thing even though we also need to take account of warnings that are issues about some of the potential dangers of AI to society [10]. We believe in particular that there are many areas with great potential to further improve personalized health care. The combination of AI and IoT (Internet of Things) looks particularly interesting. Apart from social robots which we discussed in this paper, more and more other devices such as wearables (e.g., smart watches) and other sensors for monitoring individual health status (e.g., for fall detection, sleeping behavior) will become available and connected to the Internet. This means that more and more health care data will become available that can be analyzed using AI techniques with the aim of improving care and detecting health issues early. The potential of AI in combination with advanced sensor technologies and computing power in the cloud is huge. In order to benefit from this potential at the individual patient level, we call for more research into Social AI and personalization techniques in particular.

\section{Bibliography}

1. Damásio AR. Descartes' Error - Emotion, Reason and the Human Brain, Grosset/Putnam Press, New York (1994).

2. Dastani M., et al. "A Practical Agent Programming Language", in: Programming Multi-Agent Systems (Proc. ProMAS 2007) (M. Dastani, A. El Fallah Seghrouchni, A. Ricci and M. Winikoff, eds.), LNAI 4908, Springer, Berlin/Heidelberg (2008): 107123.

3. Arnab S., et al. Serious Games for Healthcare: Applications and Implications, IGI Global, Hershey, Pennsylvania, USA (2012).

4. Boumans R., et al. "Robot for health data acquisition among older adults: a pilot randomised controlled cross-over trial". BMJ Quality and Safety (2019).

5. Boumans R., et al. "A Feasibility Study of a Social Robot Collecting Patient Reported Outcome Measurements from Older Adults". International Journal of Social Robotics (2019): 1-8.

6. Davis R,. et al. "The Origin of Rule-Based Systems in AI". reprinted as Ch. 2 of Rule Based Expert Systems: The Mycin Experiments of the Stanford Heuristic Programming Project (The Addison-Wesley Series in Artificial Intelligence). Bruce G. Buchanan and Edward H. Shortliffe (Eds.). Addison-Wesley Longman Publishing Co., Inc., Boston, MA, USA (1984).

7. Eysenbach G. "Health Care Robotics: Qualitative Exploration of Key Challenges and Future Directions". Journal of Medical Internet Research 20.7 (2018).

8. Frijda NH. "Emotions and Action", Ch 10 of: Feelings and Emotions: The Amsterdam Symposium (Antony S. R. Manstead, Nico Frijda, Agne.a Fischer (eds.)), Cambridge Univ. Press, Cambridge, UK (2004).

9. Groot P., et al. "Adaptation of clinical practice guidelines, Computer-based Medical Guidelines and Protocols". (2008): 121139.

10. Hawking S. "Stephen Hawking says A.I. could be 'worst event in the history of our civilization'”, (2017).

11. Henkemans OAB., et al. "Using a robot to personalise health education for children with diabetes type 1: A pilot study". Patient education and counseling 92 (2) (2013): 174-181.

12. Henkemans OAB., et al. "Design and evaluation of a personal robot playing a self-management education game with chil- 
dren with diabetes type 1". International Journal of HumanComputer Studies 106 (2017): 63-76.

13. Hindriks KV., et al. "An interview robot for collecting patient data in a hospital". Ercim News 114 (2018).

14. Höhle D., et al. Classifying the Decision to Perform Surgery in MEN1 Cancer Patients Using Decision Trees, in Proc. CBMS 2011 (the 24th Int. Symp. on Computer-Based Medical Systems) and HEALTHGRID 2011, (T. Solomonides, ed.), Bristol (2011).

15. Horsch C., et al. "Towards the usage of persuasive strategies in a virtual sleep coach". In proceedings of UKHCI 2012 Workshop on People, Computers and Psychotherapy (2012).

16. Horsch $\mathrm{CH}$., et al. "Mobile phone-delivered cognitive behavioral therapy for insomnia: a randomized waitlist controlled trial”. Journal of medical Internet research 19.4 (2017): e70.

17. Jones AJI., et al. "The design of intelligent socio-technical systems”. Artificial Intelligence Review 39.1 (2013): 5-20.

18. Kaptein F., et al. "The role of emotion in self-explanations by cognitive agents". Seventh International Conference on Affective Computing and Intelligent Interaction Workshops and Demos (ACIIW). IEEE. (2017): 88-93.

19. Kayal A., et al. "Socially adaptive electronic partners for improved support of children's values: An empirical study with a location-sharing mobile app". International Journal of ChildComputer Interaction 18 (2018).

20. Larochelle H., et al. "Classification Using Discriminative Restricted Boltzmann Machines". Proceedings of the 25th international conference on Machine learning - ICML '08 (2008): 536.

21. Lartigue M. "TEDx How can robotics and A.I. help children with autism?". (2019).

22. Ligthart M., et al. "Reducing stress by bonding with a social robot: Towards autonomous long-term child-robot interaction". Companion of the 2018 ACM/IEEE International Conference on Human-Robot Interaction. ACM (2018): 305-306.

23. Ligthart M., et al. "A Child and a Robot Getting AcquaintedInteraction Design for Eliciting Self-Disclosure". Proceedings of the 18th International Conference on Autonomous Agents and MultiAgent Systems. International Foundation for Auton- omous Agents and Multiagent Systems (2019): 61-70.

24. Lindley DV. "Regression and correlation analysis", New Palgrave: A Dictionary of Economics, 4 (1987): 120-23.

25. Lloyd SP. "Least squares quantization in PCM". IEEE Transactions on Information Theory 28.2 (1982): 129-137.

26. van der Lubbe LM., et al. "A serious game for training verbal resilience to doorstep scams". In: International Conference on Games and Learning Alliance. Springer, Cham. (2018): 110120.

27. van der Lubbe LM., et al. "An application for training verbal resilience to doorstep scams using virtual agents". In: Atzmueller, M.; Duivesteijn, W.(ed.), BNAIC'18: Proceedings of the 30th Benelux Conference on Artificial Intelligence (2018): 125-126.

28. van der Lubbe LM., et al. "Designing a system with persuasive communication to improve diet compliance for elderly users". In: Proceedings of the 13th EAI International Conference on Pervasive Computing Technologies for Healthcare. ACM (2019): 234-241. ACM.

29. “The Top 12 Social Companion Robots". The Medical Futurist (2018).

30. Meyer JJC. "Reasoning about Emotional Agents". in Proc.16th European Conf. on Artif. Intell. (ECAI 2004) (R. López de Mántaras and L. Saitta, eds.), IOS Press (2004): 129-133; full version in Int. J. of Intelligent Systems 21.6 (2006): 601-619.

31. Meyer JJC, "Agent Technology", in: Encyclopedia of Computer Science and Engineering 1 (B.W. Wah, ed.), Wiley, Hoboken, NJ (2009): 42-49.

32. Meyer JJC., et al. "A Logical Approach to the Dynamics of Commitments". Artificial Intelligence 113 (1999): 1-40.

33. Mols B., et al. Hallo Robot: Meet Your New Workmate and Friend, Canbury Press (2018).

34. Montemerlo M., et al. "Experiences with a Mobile Robotic Guide for the Elderly". Proc. 18th National Conference on Artificial Intelligence (AAAI), AAAI Press (2002): 587-592.

35. Oatley K., et al. Understanding Emotions, Blackwell Publishing, Malden/Oxford (1996). 
36. Ortony A., et al. The Cognitive Structure of Emotions, Cambridge University Press, Cambridge (1988).

37. Pearl J. "Bayesian Networks: A Model of Self-Activated Memory for Evidential Reasoning". Proceedings of the 7th Conference of the Cognitive Science Society, University of California, Irvine, CA. (1985): 329-334.

38. Picard RW. "Affective Computing". MIT Press (1997).

39. Pineau J., et al. "Towards Robotic Assistants in Nursing Homes: Challenges and Results". Robotics and Autonomous Systems 42.3-4 (2003): 271-281.

40. Quinlan JR. "Induction of decision trees". Machine Learning 1 (1986): 81-106.

41. Reisenzein R., et al. "Computational Modeling of Emotion: Toward Improving the Inter- and Intradisciplinary Exchange". IEEE Transactions on Affective Computing 4.3 (2013): 246266.

42. Robins B., et al. "Developing interaction scenarios with a humanoid robot to encourage visual perspective taking skills in children with autism, preliminary proof of concept tests". In International Conference on Social Robotics. Springer, Cham (2017): 147-155.

43. Shannon CE. "A Mathematical Theory of Communication". The Bell System Technical Journal 27 (1948): 379-423 and 623656.

44. Shakshukia E., et al. "Multi-Agent System Applications in Healthcare: Current Technology and Future Roadmap". Proc. The 6th International Conference on Ambient Systems, Networks and Technologies (ANT 2015), Procedia Computer Science 52, Elsevier (2015): 252-261.

45. Spekman MLC. The Role of Emotion in the Study of Humanoid Social Robots in the Healthcare Domain, PhD Thesis, VU University, Amsterdam (2018).

46. Spekman MLC., et al. "Perceptions of healthcare robots as a function of emotion-based coping: The importance of coping appraisals and coping strategies". Computers in Human Behavior 85 (2018): 308-318.

47. Steels L., et al. The Artificial Life Route to Artificial Intelligence: Building Situated Embodied Agents, Lawrence Erlbaum Ass., New Haven (1994).
48. Steunebrink BR., et al. "Emotions as Heuristics in Multi-Agent Systems", in: D. Reichhardt, P. Levi and J.-J. Ch. Meyer (eds.), Proc. 1st Workshop on Emotion and Computing - Current Research and Future Impact, held at KI2006, Bremen (2006): 15-18.

49. Steunebrink BR., et al. "A Logic of Emotions for Intelligent Agents", in Proc. AAAI-07 (R.C. Holte and A.E. Howe, eds.), Vancouver, Canada, AAAI Press (2007): 142-147.

50. Steunebrink BR., et al. "A Formal Model of Emotion-Based Action Tendency for Intelligent Agents". in: Progress in Artificial Intelligence (14th Portuguese Conference on Artificial Intelligence, EPIA 2009) (L. Seabra Lopes, N. Lau, P. Mariano, L.M. Rocha, eds.), Aveiro, Portugal, LNAI 5816, Springer, Berlin/ Heidelberg (2009): 174-186.

51. Steunebrink BR., et al. "Emotions to Control Agent Deliberation". Proc. of 9th Int. Conf. on Autonomous Agents and Multiagent Systems (AAMAS 2010) (W. van der Hoek, G. Kaminka, Y. Lespérance, M. Luck and S. Sen, eds.), Toronto, Canada, IFAAMAS (2010): 973-980.

52. Steunebrink BR., et al. "A Formal Model of Emotion Triggers for BDI Agents with Achievement Goals". Synthese/KRA 185.1 (2012): 83-129 (KRA, 413-459).

53. Ten Teije A., et al. "Computer-based medical guidelines and protocols: a primer and current trends". IOS Press (2008).

54. Ten Teije A., et al. "Improving medical protocols by formal methods". Artificial Intelligence in Medicine 36.3 (2006): 193209.

55. Wood L., et al. "Developing child-robot interaction scenarios with a humanoid robot to assist children with autism in developing visual perspective taking skills". In: 26th IEEE International Symposium on Robot and Human Interactive Communication (RO-MAN), IEEE (2017): 1055-1060.

56. Woolf SH. "Evidence-based medicine and practice guidelines: an overview". Cancer Control: Journal of the Moffitt Cancer Center 7.4 (2000): 362-367.

\section{Volume 3 Issue 10 October 2019 (c) All rights are reserved by Koen $V$ Hindriks and John-Jules Ch Meyer.}

